\title{
Crossed Testicular Ectopia
}

\author{
Anup Pradhan, ${ }^{1}$ Om Biju Panta, ${ }^{2}$ Ghanshyam Gurung, ${ }^{2}$ Dan Bahadur Karki ${ }^{3}$ \\ 'Kist Medical College and Hospital, Lalitpur,Nepal, ${ }^{2}$ Tribhuwan University Teaching Hospital, Kathmandu, ${ }^{3}$ Patan Academy \\ of Health Sciences, Lalitpur, Nepal.
}

\section{ABSTRACT}

Crossed testicular ectopia also known as transverse testicular ectopia is a rare anomaly of the testis which is ectopically located in contralateral hemiscrotum with absent testis in the ipsilateral hemiscrotum. Most case are incidentally discovered intraopeartively during operation of inguinal hernia and few case reports are available which have reported preoperative diagnosis of crossed testicular ectopia. We report a case of crossed testicular ectopia in 12 year old boy who presented with right undescended testis diagnosed preoperative on Ultrasound and Magnetic Resonance Imaging as crossed testicular ectopia.

Keywords: crossed ectopia; MRI; testis; ultrasonography.

\section{INTRODUCTION}

Crossed testicular ectopia also known as transverse testicular ectopia is rare congenital testicular migrational abnormality in which both testicles migrate to one hemiscrotum. The displaced testis may lie in the contralateral inguinal canal, deep inguinal ring or contralateral hemiscrotum. Clinically it may be suspected when one scrotal sac is empty or symptomatic inguinal hernia on one side to which ectopic gonad has migrated. In most cases, the diagnosis of crossed ectopic testis is made during surgical exploration for inguinal hernia. However imaging studies provide an insight to the other associated anomalies and are more useful then laproscopic examination. ${ }^{1}$ Hereby, we present a case which had been prediagnosed as having right undescended testicle and was diagnosed with ultrasound and MRI as crossed ectopic testis.

\section{CASE REPORT}

A 12 year old boy came to our center with c/o non palpable right testicle for imaging studies with provisional diagnosis of an undescended right testicle. Family history was insignificant. On physical examination, right scrotum was empty and the right testis could not be palpable on scrotum or inguinal canal or pelvic region with normal appearing urethral meatus. Left testis was palpable in left scrotum. Ultrasonography was performed with linear probe and revealed right scrotum was empty. Ipsilateral inguinal canal was examined but there was no evidence of undescended testis. Both testis were found in left scrotum (Figure 1). The size of both testis were symmetrical and both testis were normal in outline with parenchymal echotexture and

Correspondence: Dr. Om Biju Panta, Department of Radiology and Imaging, Tribhuwan University Teaching Hospital, Kathmandu, Nepal. Email: bijupanta@yahoo.com, Phone: +977-9842877382. 
echogenecity. Using conventional spin-echo sequences MRI was performed. MRI revealed empty right scrotum and double testis into left hemiscrotum. It pointed out the position of two testis and both the testes were normal in size, shape and signal intensity (Figure 2). The patient was diagnosed as crossed testicular ectopia and was referred to surgery department.

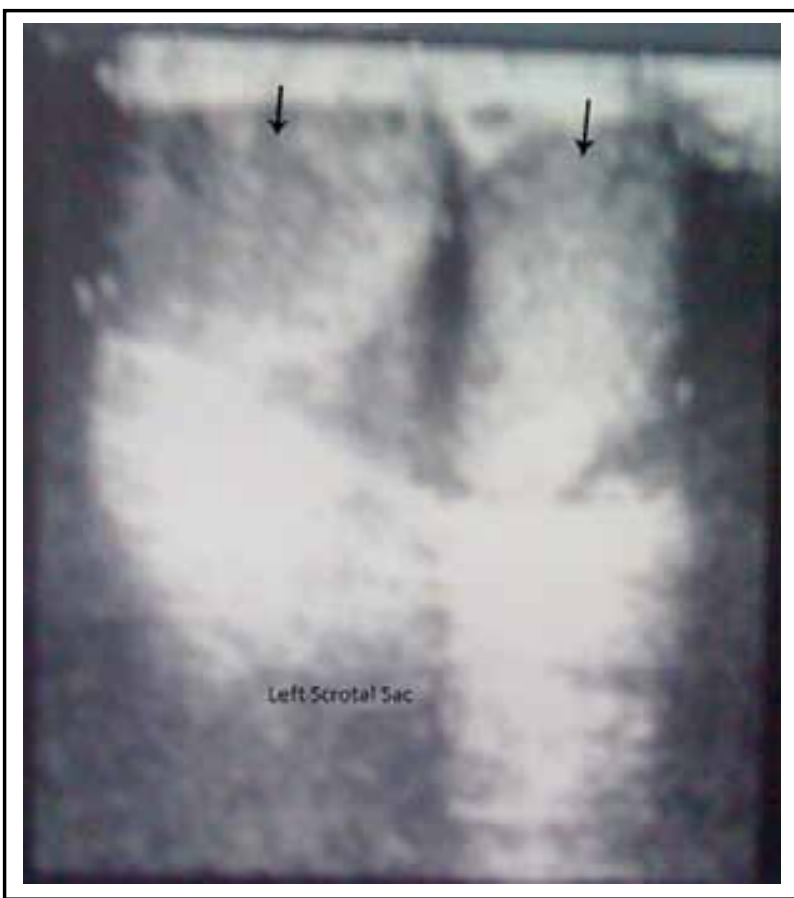

Figure 1. Ultrasonography image showing two testis located in left hemiscrotum.

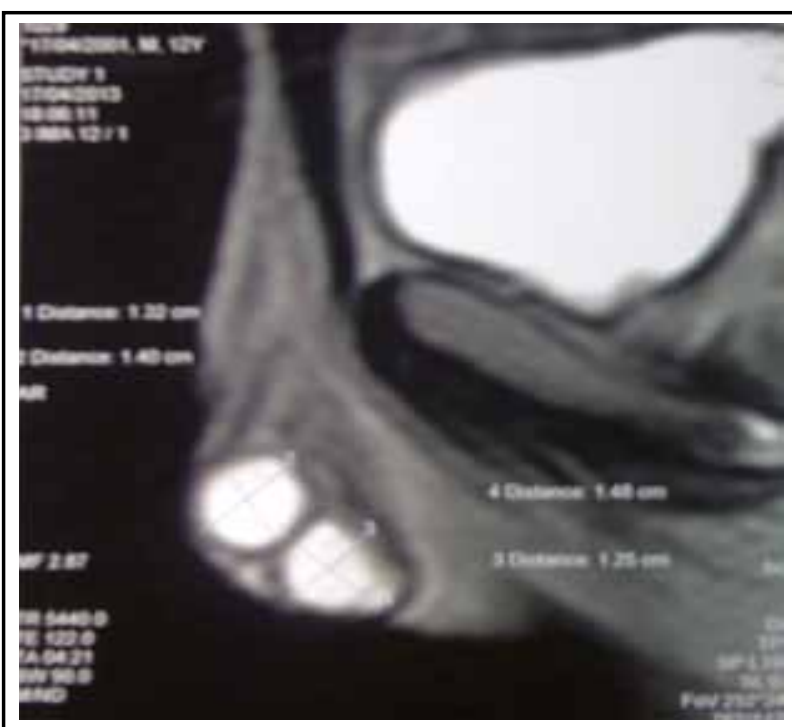

Figure 2. Crossed ectopia detected in MRI. Sagittal T2 weighted image shows maldescent of right testis in left hemiscrotum. Both the testis are of same size and same signal intensity.

\section{DISCUSSION}

The testis forms in the primitive genital ridge medial to the mesonephric duct in the abdominal cavity. The testes due to inteplay of various factors : the gubernaculum, the increase in abdominal pressure and the neurohormonal factors, descent into the inguinal canal then to the scrotum. ${ }^{2}$ In around $97 \%$ of the male child the testes are found in scrotum at birth; and in most of the remainder the testes are descended into the scrotum by three month of age. In about one percent the testes fail to descend to the scrotum which is termed as crptoorchidism. ${ }^{3}$ The testes may sometime maldescent and ectopic testes have been reported in different sites, including suprapubic, femoral area, perineal area, the base of the penis.

Crossed testicular ectopia is a very rare form of ectopic testis. The testis of both side is located in the same hemiscrotum with empty contralateral hemiscrotum. The embryological basis for crossed ectopic testis is not totally understood, however various theories have been proposed in an attempt to explain the maldescent of the testis. Lenhossek in 1886 first described crossed ectopic tested and proposed abnormality in gumernaculum to be the culprit for the same. ${ }^{4}$ Abnormality in gubernaculums has also been supported by Lockwood in 1888 and Josso in $1977 . .^{5}$ Some other consider the abnormality as a result of origin of bilateral testis from a single genital ridge ( Berg-1905) while other debate the presence of two separate deferent duct to indicate origin from separate genital ridge. Kimura et al. considered true crossed ectopic testis only if two separate deferent ducts are present, a common duct suggesting the development of the testis from one genital ridge. ${ }^{6}$ Obstruction of the inguinal canal, adherences between the testis and the nearby structures, adhesion of wolffian duct and pull by the persistent paramesonephric duct are other proposed theories. ${ }^{5}$ Due to histologic similarities between ectopic and undescended testes some consider them as a spectrum of same disease. ${ }^{7}$

Crossed testicular ectopia is commonly associated with other anomalies of genitourinary tract. ${ }^{5}$ According to its association, crossed testicular ectopia has been classified as 1) associated with inguinal hernia or persistent processus vaginalis which accounts upto $50 \%$ of cases, 2) associated with persistence or rudimentary structures of mullerian duct (30\%) and 3) associated with anomalies other than inguinal hernia or persistence of the müllerian duct, such as pseudohermaphroditism, inguinal hernias, hypospadias, and scrotal anomalies $(20 \%)^{8}$

Most patients present to the clinician for the investigation of an undescended testes and are 
diagnosed with crossed testicular ectopia during the operation for the hernia or rarely on imaging studies. Often the right testes is ectopically located and is associated with contralateral inguinal hernia which may also incarcerate at presentation. ${ }^{1}$ However clinically a crossed testicular ectopia should be suspected in a patient with undescended testes and contra lateral inguinal hernia as it is the commonest association.

Ultrasound due to its versatility and lack of radiation remains the first investigation for investigating an undescended testes and crossed testicular ectopia as well especially in children. ${ }^{9}$ Occasionally when the diagnosis is missed on ultrasound and testes is not visualized MRI should be performed to search for the non-visualized gonad. MRI and MR angiography are also recommended techniques for preoperative diagnosis of crossed testicular ectopia. MR venogram is especially important in vanishing testis when the testicular tissue are not visualized by ultrasound or MRI. ${ }^{9}$ On MRI crossed testicular ectopia classically is seen as characteristic bright signal on T2-W images and a linear low-signal structure which may represent the remnant of the gubernaculum testis with both testis on same hemiscrotum or ipsilateral ingunal canal with empty contralateral hemiscrotum. ${ }^{9}$

Once the diagnosis of crossed testicular ectopia is made it is important to look for other genitouninary anomalies especially the persistence of mullerian duct in these patients. Also patients with transtesticualr ectopia may be at an increased risk of malignant testicular mass ( $5 \%$ risk), so these patients need continuous long term follow up and scrutiny for any malignant masses. ${ }^{5}$

\section{REFERENCES}

1. Cece H, Abuhandan M, Yildiz S, Karakas O, Karakas E. A Case Presentation With Tranverse Testicular Ectopia. Int J ClinPediatr. 2012;1(1):49-51.

2. Husmann DA, Levy JB. Current concepts in the pathophysiology of testicular undescent. Urology. 1995;46(2):267-76.

3. Sadler TW. Langman's medical embryology. $12^{\text {th }}$ ed. USA:Lippincott Williams \& Wilkins;2011.

4. Von Lenhossek MN. Ectopia testis transversa. Anat Anz. 1886;1:376-81.

5. Basca I, Nica A, Niculescu L, Andriescu M, Datu C, Basca I. Crossed Testicular Ectopia. Journal of Pediatric Surgical Specialties. 2010;4(1):1-66.
6. Kimura T. Transverse ectopy of the testis with masculine uterus. Ann Surg. 1918;68(4):420-5.

7. Hutcheson JC, Snyder HM, Zuniga ZV, Zderic SA, Schultz DJ, Canning DA, et al. Ectopic and undescended testes: 2 variants of a single congenital anomaly? J Urol. 2000;163(3):961-3.

8. Pinto CW, Toro RC, Mansilla KH, Castro TA, Fuente-Alba CS. Crossed testicular ectopia. Journal of Ultrasound in Medicine. 2010;29(5):847-9.

9. Lam WW, Le SD, Chan KL, Chan FL, Tam PK. Transverse testicular ectopia detected by MR imaging and MR venography. Pediatr Radiol. 2002;32(2):126-9. 